\title{
More severe clinical course of cardiovascular dysfunction in intensive care unit patients with the 894TT eNOS genotype
}

L.R. Fraga ${ }^{1,2}$, F.J.O. Paludo ${ }^{1,3}$, A.J.G. Bós ${ }^{4}$, J.L.S. Ferraro ${ }^{1}$, F.S. Dias ${ }^{5}$ and C.S. Alho ${ }^{1}$

${ }^{1}$ Faculdade de Biociências, Pontifícia Universidade Católica do Rio Grande do Sul, Porto Alegre, RS, Brasil

${ }^{2}$ Departamento de Genética, Instituto de Biociências, Universidade Federal do Rio Grande do Sul, Porto Alegre, RS, Brasil ${ }^{3}$ Departamento de Bioquímica, Instituto de Ciências Básicas da Saúde, Universidade Federal do Rio Grande do Sul, Porto Alegre, RS, Brasil ${ }^{4}$ Instituto de Geriatria e Gerontologia,

Pontifícia Universidade Católica do Rio Grande do Sul, Porto Alegre, RS, Brasil

${ }^{5}$ Hospital São Lucas, Unidade de Terapia Intensiva, Porto Alegre, RS, Brasil

Corresponding author: C.S. Alho

E-mail: csalho@pucrs.br

Genet. Mol. Res. 12 (1): 562-568 (2013)

Received May 14, 2012

Accepted October 30, 2012

Published February 27, 2013

DOI http://dx.doi.org/10.4238/2013.February.27.5

ABSTRACT. The endothelial nitric oxide synthase (eNOS) plays an important homeostatic role in the cardiovascular system (CVS) by maintaining appropriate blood pressure through production of nitric oxide. The 894TT genotype of 894G>T (Glu298Asp, rs1799983), a polymorphic variant of $e N O S$, has been associated with several vascular diseases. On the basis of this strong relationship, we monitored daily 585 critically ill adult patients according to their degree of CVS dysfunction and investigated their disease progression by the $894 \mathrm{G}>\mathrm{T}$ genotype. To obtain information of the general population, we obtained the $894 \mathrm{G}>\mathrm{T}$ 
genotypic and allelic frequencies in a random group of 149 healthy subjects. The patients were genotyped for the eNOS $894 \mathrm{G}>\mathrm{T}$ polymorphism and daily evaluated according to their degree of CVS dysfunction through the Cardiovascular Sequential Organ Failure Assessment (SOFA) score. The mean value of the global CVS dysfunction score was significantly higher in 894TT patients $(1.35 \pm 0.57)$ than in non-894TT patients $(1.23$ $\pm 0.37 ; \mathrm{P}=0.035$ ). This score remained significantly higher in 894TT patients, even in different patient clusters (all patients, septic, and nonseptic patients) during the 1 st week at the intensive care unit $(1.86 \pm 0.8$ versus $1.63 \pm 0.62, \mathrm{P}=0.005 ; 2.32 \pm 0.10$ versus $2.06 \pm 0.08, \mathrm{P}=0.009$; $0.84 \pm 0.09$ versus $0.64 \pm 0.08, \mathrm{P}=0.027$; respectively). This result shows that the mean values of the cardiovascular SOFA score were higher in 894TT patients in all subgroups. The present study provides evidence that the 894TT eNOS genotype is associated with a higher degree of CVS dysfunction in critically ill patients.

Key words: eNOS; 894G>T SNP; Genetic risk factors; SOFA score; Cardiovascular system dysfunction; Critically ill patients

\section{INTRODUCTION}

The endothelial nitric oxide synthase (eNOS) plays an important homeostatic role in the cardiovascular system (CVS) by relaxing the endothelium and maintaining appropriate blood pressure through production of nitric oxide (Moncada and Higgs, 1993; Umans and Levi, 1995). Within exon 7 of the eNOS gene, there is a single nucleotide polymorphism (SNP) that replaces the 894 guanine to thymine $(894 \mathrm{G}>\mathrm{T}$; rs1799983), resulting in amino acid replacement at position 298 (Glu298Asp) (Nadaud et al., 1994; Miyamoto et al., 1998). The 894T allele is associated with a reduction in the function of the enzyme (Tesauro et al., 2000), and several studies have addressed positive relationships between the 894TT genotype and vascular disease states such as coronary artery disease (Hingorani et al., 1999; Kerkeni et al., 2006), hypertension (Miyamoto et al., 1998), myocardial infarction (Hibi et al., 1998; Antoniades et al., 2005), and atherosclerosis (Lembo et al., 2001). On the basis of the strong relationship between this eNOS polymorphism and the function of the CVS, we monitored daily 585 critically ill adult patients according to their degree of CVS dysfunction (maximum duration of monitoring: 199 days) and investigated disease progression by the $894 \mathrm{G}>\mathrm{T}$ genotype.

\section{MATERIAL AND METHODS}

In this study, we included a total of 585 (304 men and 281 women) critically ill adult patients from the general intensive care unit (ICU) at the São Lucas Hospital, Porto Alegre, RS, Brazil, admitted between January 2003 and December 2007. The patients were monitored from their admission to the ICU until discharge from the hospital or death, for a maximum of 199 days. They were daily evaluated according to their degree of CVS dysfunction. In addition, to determine the allele frequencies in the general population, we collected data from a random group of 149 healthy subjects. All subjects (patients and healthy donors) were from 
southern Brazil, which is composed of a singular genetic background: the majority of subjects were of European origin (Portuguese, Italians, Spanish, and Germans ancestry), and there was a small number of individuals with African traits contributing to their genetic pool (Parra et al., 2003). This study was approved by the Ethics Committee of our institute (Protocol \#05/02598), and informed consent was obtained from all participants.

\section{Phenotyping}

We evaluated daily the arterial pressure and the necessity of administration of dopamine, dobutamine, adrenaline, or noradrenaline to normalize the abnormal arterial pressure. We transformed these data to obtain the Cardiovascular Sequential Organ Failure Assessment (SOFA; Vincent et al., 1998, Ferreira et al., 2001) scores as follows: score $0=$ mean arterial pressure (MAP) $\geq 70 \mathrm{mmHg}$; score $1=\mathrm{MAP}<70 \mathrm{mmHg}$; score 2 = necessity of administration of dopamine or dobutamine $(\leq 5 \mathrm{mg})$; score $3=$ necessity of administration of dopamine $(>5 \mathrm{mg})$, or adrenaline $(\leq 0.1 \mathrm{mg})$ or noradrenaline $(\leq 0.1 \mathrm{mg})$, and score $4=$ necessity of administration of dopamine $(>15 \mathrm{mg})$, adrenaline $(>0.1 \mathrm{mg})$, or noradrenaline $(>0.1 \mathrm{mg})$. The cardiovascular SOFA score was evaluated in all 585 patients during the entire stay at the ICU. For the evaluation of the severity of general illness, we used the Acute Physiology and Chronic Healthy Evaluation II (APACHE-II; Knaus et al., 1985) score obtained on the day of ICU admission. Mortality was measured in days until death. For the diagnosis of sepsis and septic shock, we used the American College of Chest Physicians/Society of Critical Care Medicine Consensus Conference criteria (Levy et al., 2003). Different phenotypic and genotypic information about these patients was found in Paskulin et al. (2011), Paludo et al. (2013), and Fallavena et al. (2013).

\section{Genotyping}

Genomic DNA was isolated from leukocytes according to Lahiri and Nurnberger Jr. (1991) and we followed the 894G $>$ T genotyping protocol described by Hibi et al. (1998). We used a quality control system to ensure genotyping accuracy: sequencing verification of the amplified DNA fragment, black controls, and repetitions. Thus, in order to confirm that the product was correctly amplified by polymerase chain reaction, we performed a sequence analysis on the MegaBace 1000 capillary DNA sequencer (Amersham Biosciences UK Ltd., Chalfont St. Giles, Bucks, UK). Alignment view was performed using the ClustalX program (version 1.8, as described at ftp://ftp-igbmc.u-strasbg.fr/pub/ClustalX/) in multiple alignment modes, with sequences uploaded in the FASTA format. The sequence obtained was submitted to a nucleotide-nucleotide BLAST online alignment (http://www.ncbi.nlm.nih.gov/BLAST/) with the human genome and we found consensus with Homo sapiens nitric oxide synthase 3 (endothelial cell: NOS3; GI:48762674, NM_000603). At least 20\% of all samples were confirmed by a 2 nd and independent genotyping. According to HapMap, the expected prevalence of the $894 \mathrm{G}>\mathrm{T}$ SNP was sufficient in our patient population: Caucasian/European heritage $894 \mathrm{~T}=0.34,894 \mathrm{G}=0.66$ (http://www.hapmap.org/). These frequencies were compatible with a previous study performed in our population with 156 cardiac patients $(894 \mathrm{~T}=0.36$ and $894 \mathrm{G}=0.64$; Iturry-Yamamoto et al., 2007). The allele frequencies in our control population (healthy individuals) were as follows: $894 \mathrm{TT}=0.31,894 \mathrm{GT}=0.33$, and $894 \mathrm{GG}=0.36$; $894 \mathrm{~T}$ 
$=0.48$ and $894 \mathrm{G}=0.52$; chi-square test Hardy-Weinberg equilibrium: $\mathrm{P}=0.099$. We did not consider healthy subjects as the control group because we assumed that environmental factors had a crucial influence. Genotyping was performed in a blinded fashion, i.e., investigators were unaware of patient data.

\section{Statistical analysis}

Statistical analysis was performed using SPSS (Chicago, IL, USA) for Windows (version 14.1, Copyright SPSS Inc.). The multilevel linear regression model was used to evaluate the progression of CVS dysfunction. The number of days that each patient remained at the ICU during these 2 weeks was introduced as random effect. Genotypes were introduced in the model as fixed-effect to detect differences between genotypes, independent of the observation time. Differences in the degree of CVS dysfunction of each patient were introduced in the test as dependent variables. Non-normally distributed variables were analyzed by the Student $t$-test and the Mann-Whitney U-test. For categorical data, we used the Pearson chi-squared test. To test Hardy-Weinberg equilibrium, the chi-squared test was used. A P value $<0.05$ was considered to indicate statistical significance.

\section{RESULTS}

Demographic and clinical characteristics of the patients by genotype group are shown in Table 1. The genotypic and allelic frequencies were: $894 \mathrm{TT}=0.11,894 \mathrm{GT}=0.42,894 \mathrm{GG}$ $=0.47,894 \mathrm{~T}=0.32$, and $894 \mathrm{G}=0.68$; these allele and genotype frequencies did not differ significantly from those predicted by the Hardy-Weinberg distribution (chi-square test HardyWeinberg equilibrium: $\mathrm{P}=0.572$ ). For genotype analysis, a recessive heredity model to $894 \mathrm{~T}$, the rarest allele, was assumed (combined 894TT versus the 894GT+894GG genotypes).

\begin{tabular}{|c|c|c|c|c|}
\hline Characteristic & All $(\mathrm{N}=585)$ & $894 \mathrm{TT}(\mathrm{N}=67)$ & $894 \mathrm{GT}+\mathrm{GG}(\mathrm{N}=518)$ & $\mathrm{P}$ \\
\hline Frequency $[\mathrm{N}(\%)]$ & $585(100)$ & $67(11.5)$ & $518(88.5)$ & $0.572^{\mathrm{HW}}$ \\
\hline Female $[\mathrm{N}(\%)]$ & $281(48)$ & $29(43.3)$ & $252(48.6)$ & $0.408^{x^{2}}$ \\
\hline Age [mean (SD)] & $55.4(19.8)$ & $58.1(19.4)$ & $55.1(19.8)$ & $0.236^{\mathrm{ST}}$ \\
\hline APACHE-II score [mean (SD)] & $19.7(7.8)$ & $20.9(7.6)$ & $19.6(7.8)$ & $0.175^{\mathrm{ST}}$ \\
\hline Global Cardiovascular score [mean (SD) $]^{\mathrm{a}}$ & $0.95(1.5)$ & $1.35(0.57)$ & $1.23(0.37)$ & $0.035^{\mathrm{MR} *}$ \\
\hline Sepsis $[N(\%)]$ & $413(70.6)$ & $47(70.1)$ & $366(70.7)$ & $0.932^{x^{2}}$ \\
\hline Septic shock $[N(\%)]$ & $291(49.7)$ & $37(55.2)$ & $254(49.0)$ & $0.340^{x^{2}}$ \\
\hline ICU LOS [median $(\min / \max )]$ & $14(0 / 125)$ & $15(2 / 78)$ & $13(0 / 125)$ & $0.218^{\mathrm{MW}}$ \\
\hline ICU+H LOS [median $(\min / \max )]$ & $36(1 / 242)$ & $40(7 / 108)$ & $35(1 / 242)$ & $0.448^{\mathrm{MW}}$ \\
\hline Mortality in ICU [N (\%)] & $190(32.5)$ & $27(40.3)$ & $163(31.5)$ & $0.146^{x^{2}}$ \\
\hline Mortality in ICU+H [N (\%)] & $264(45.1)$ & $33(49.3)$ & $231(44.9)$ & $0.496^{x^{2}}$ \\
\hline
\end{tabular}

aPatients' cardiovascular score values measured by sequential organ failure assessment. $894 \mathrm{GT}+\mathrm{GG}=$ heterozygotes; 894TT = homozygotes; APACHE-II = Acute Physiology and Chronic Health Evaluation II; ICU = intensive care unit; ICU+H = ICU plus hospital; LOS = length of stay; $\mathrm{N}=$ number; $\mathrm{SD}=$ standard deviation; HW = Pearson chi-square test for Hardy-Weinberg equilibrium; $\chi^{2}=$ Pearson chi-square test; ST $=$ Student $t$-test; MW $=$ MannWhitney $U$-test; MR = multi-level linear regression model test; ${ }^{*} \mathrm{P}$ value describes a comparison between $894 \mathrm{TT}$ versus no 894TT genotype groups.

There was no significant difference between the genotype groups (894TT versus 
894GT + 894GG patients) and the patient characteristics, i.e., gender, age, APACHE-II score, occurrence of sepsis or septic shock, length of stay at the ICU and post-ICU (hospital), and mortality rates. However, the mean value of the global cardiovascular dysfunction score was significantly higher in $894 \mathrm{TT}$ patients $(\mathrm{P}=0.035)$. Table 2 presents a discriminated analysis of the effect of the 894TT genotype in different patient clusters (all patients, septic, and non-septic patients) during the 1 st week at the $\operatorname{ICU}(\mathrm{P}=0.005, \mathrm{P}=0.009$, and $\mathrm{P}=0.027$, respectively). In all subgroups, the mean values of the cardiovascular SOFA scores were higher in 894TT patients, but the 894TT effect over CVS dysfunction was most decisive and significant during the 1 st week at the ICU. There was no significant difference among the subgroups in the following ICU weeks when analyzed separately. The 894TT genotype influenced the patients' CVS dysfunction independently; even in patients with sepsis and septic shock, the degree of CVS dysfunction was significantly higher in 894TT patients.

\begin{tabular}{|c|c|c|c|c|}
\hline & Patients & 894TT $(\mathrm{N}=67)$ & $894 \mathrm{GT}+\mathrm{GG}(\mathrm{N}=518)$ & $P$ \\
\hline All patients [mean (SD)] & $1.12(1.6)$ & $1.86(0.80)$ & $1.63(0.62)$ & $0.005^{\mathrm{MR} *}$ \\
\hline Patients with sepsis [mean (SD)] & $1.38(1.7)$ & $2.32(0.10)$ & $2.06(0.08)$ & $0.009^{\mathrm{MR} *}$ \\
\hline Patients without sepsis [mean (SD)] & $0.41(1.1)$ & $0.84(0.09)$ & $0.64(0.08)$ & $0.027^{\mathrm{MR} *}$ \\
\hline
\end{tabular}

\section{DISCUSSION}

Here, we found that the degree of CVS dysfunction was significantly higher in 894TT patients when compared with 894GT+894GG patients during the crucial 1st week at the ICU. This finding provides new information about the effect of the $894 \mathrm{G}>\mathrm{T}$ polymorphism on the condition of the CVS, suggesting that the 894T variant in homozygous subjects has a negative effect on CVS function when the patient is under critical circumstances.

The present study used the multilevel linear regression model, also called mixed-effect linear model, which is an appropriate model for analyzing longitudinal data to repeated measures of the same patient. The model consists of an analysis that is expressed as a function of both components: fixed (overall prediction) and random (inherited individual predictions). We grouped covariates according to a classification factor (the $894 \mathrm{G}>\mathrm{T}$ genotype, fixed-effect) and the time between measurements (random-effect) with a continuous dependent variable (CVS dysfunction daily scores). This longitudinal approach was helpful in understanding how subtle was the association between a factor (genotype) and a variable that change over the time.

The $894 \mathrm{~T}$ allele is associated with worse eNOS functionality, i.e., decreased nitric oxide production by endothelial cells, than the $894 \mathrm{G}$ variant (Veldman et al., 2002). Since eNOS is a constitutive enzyme, the entire endothelium is affected by the $894 \mathrm{G}>\mathrm{T}$ polymorphism (Veldman et al., 2002; Antoniades et al., 2005), and this directly affects the regulation of the CVS (Dafni et al., 2010). Because of this association, this common, but functional, polymorphic variation has been the focus of interest and pointed out as risk factor and/or potential marker in the pathobiology of cardiovascular diseases in different populations. Dafni et al. (2010) studied 204 patients with myocardial infarction and 218 control subjects; in that population of Greek origin, the risk of developing myocardial infarction was found to be about 
2-fold higher for 894TT patients when compared with 894G carriers. The 894TT genotype was also associated with coronary artery disease in a Tunisian population. Kerkeni et al. (2006) studied 100 patients with coronary artery disease and 120 control subjects. In addition to the effect of the 894TT genotype, the Tunisian study found a relationship between the 894T variant and hyperhomocysteinemia and severity of coronary artery disease. In another study that also evaluated the influence of this eNOS polymorphism in critically ill patients, Huttunen et al. (2009) found a significant relationship between the 894T allele and hypotension in patients with Escherichia coli bacteremia. In addition, this study, which followed the clinical course of 147 patients during the 1 st 6 days at the ICU, showed that carriers of the $894 \mathrm{~T}$ allele had lower blood pressure and higher SOFA scores. The same instrument was used by us to assess the function of the CVS in our sample population. Another finding in Huttunen's study that confirms our results is that the effect of the $894 \mathrm{~T}$ allele on blood pressure was most prominent in the early stage of the disease: on admission, MAP was lower in carriers of the 894T allele compared with non-carriers. The above mentioned studies, in addition to our study, show that this polymorphic variant affects directly the condition, structure, and proper functioning of the CVS; moreover, the allelic and genotypic frequencies resemble those found in our group.

In the vital 1st week at the ICU, the association between the 894TT genotype and worse CVS scores was powerfully exposed. Interestingly, this inherited influence gradually disappeared from the 2 nd week at the ICU by the intensive treatment. We also could note that septic patients presented the highest degree of CVS dysfunction (Table 2), independent of the genotype. Due to several physiological changes caused by the sepsis, e.g., cytokine circulation and altered homeostasis, this clinical condition causes myocardial dysfunction, affecting the CVS individually (Krishnagopalan et al., 2002). However, even in patients with sepsis, the degree of CVS dysfunction was higher in 894TT patients, indicating that the effect of this genotype is an independent factor of cardiovascular dysfunction.

In conclusion, the present study provides evidence that the 894TT eNOS genotype is associated with a higher degree of CVS dysfunction in critically ill patients. In addition, this polymorphism can be clinically useful as potent genetic marker for the prognosis of the risk to develop CVS dysfunction and of the outcome in critically ill patients.

\section{Conflict of interest}

There is no conflict of interest.

\section{ACKNOWLEDGMENTS}

Research supported by Fundação de Amparo à Pesquisa do Estado do Rio Grande do Sul - FAPERGS (EDITAL PPSUS 02/2009; process \#09/0039-3), Programa de Bolsa Pesquisa para Alunos da Graduação - BPA PUCRS 2007-2008, and Faculdade de Biociências, Pontifícia Universidade Católica do Rio Grande do Sul.

\section{REFERENCES}

Antoniades C, Tousoulis D, Vasiliadou C, Pitsavos C, et al. (2005). Genetic polymorphism on endothelial nitric oxide synthase affects endothelial activation and inflammatory response during the acute phase of myocardial infarction. 
J. Am. Coll. Cardiol. 46: 1101-1109.

Dafni C, Drakoulis N, Landt O, Panidis D, et al. (2010). Association of the eNOS E298D polymorphism and the risk of myocardial infarction in the Greek population. BMC Med. Genet. 11: 133.

Fallavena PRV, Borges TJ, Paskulin DD, Thurow HS, et al. (2013). The synergy of -260T T CD14 and -308GG TNF- $\alpha$ genotypes in survival of critically ill patients. Scandinav. J. Immunol. 77: 62-68.

Ferreira FL, Bota DP, Bross A, Melot C, et al. (2001). Serial evaluation of the SOFA score to predict outcome in critically ill patients. JAMA 286: 1754-1758.

Hibi K, Ishigami T, Tamura K, Mizushima S, et al. (1998). Endothelial nitric oxide synthase gene polymorphism and acute myocardial infarction. Hypertension 32: 521-526.

Hingorani AD, Liang CF, Fatibene J, Lyon A, et al. (1999). A common variant of the endothelial nitric oxide synthase $\left(\mathrm{Glu}^{298} \rightarrow \mathrm{Asp}\right)$ is a major risk factor for coronary artery disease in the UK. Circulation 100: 1515-1520.

Huttunen R, Hurme M, Laine J, Eklund C, et al. (2009). Endothelial nitric oxide synthase G894T (GLU298ASP) polymorphism is associated with hypotension in patients with $E$. coli bacteremia but not in bacteremia caused by a gram-positive organism. Shock 31: 448-453.

Iturry-Yamamoto GR, Moriguchi EH, Zago AC, Alho C, et al. (2007). Association of the 894G>T polymorphism of the endothelial constitutive nitric oxide synthase gene with unstable angina. Braz. J. Med. Biol. Res. 40: 475-483.

Kerkeni M, Addad F, Chauffert M, Myara A, et al. (2006). Hyperhomocysteinemia, endothelial nitric oxide synthase polymorphism, and risk of coronary artery disease. Clin. Chem. 52: 53-58.

Knaus WA, Draper EA, Wagner DP and Zimmerman JE (1985). APACHE II: a severity of disease classification system. Crit. Care Med. 13: 818-829.

Krishnagopalan S, Kumar A, Parrillo JE and Kumar A (2002). Myocardial dysfunction in the patient with sepsis. Curr. Opin. Crit. Care 8: 376-388.

Lahiri DK and Nurnberger JI Jr (1991). A rapid non-enzymatic method for the preparation of HMW DNA from blood for RFLP studies. Nucleic Acids Res. 19: 5444.

Lembo G, De Luca N, Battagli C, Iovino G, et al. (2001). A common variant of endothelial nitric oxide synthase (Glu298Asp) is an independent risk factor for carotid atherosclerosis. Stroke 32: 735-740.

Levy MM, Fink MP, Marshall JC, Abraham E, et al. (2003). 2001 SCCM/ESICM/ACCP/ATS/SIS International sepsis definitions conference. Intensive Care Med. 29: 530-538.

Miyamoto Y, Saito Y, Kajiyama N, Yoshimura M, et al. (1998). Endothelial nitric oxide synthase gene is positively associated with essential hypertension. Hypertension 32: 3-8.

Moncada S and Higgs A (1993). The L-arginine-nitric oxide pathway. N. Engl. J. Med. 329: 2002-2012.

Nadaud S, Bonnardeaux A, Lathrop M and Soubrier F (1994). Gene structure, polymorphism and mapping of the human endothelial nitric oxide synthase gene. Biochem. Biophys. Res. Commun. 198: 1027-1033.

Paludo FJO, Picanço JB, Fallavena PRV, Fraga LR, et al. (2013). Higher frequency of septic shock in septic patients with the 47C allele (rs4880) of the SOD2 gene. Gene 517: 106-111.

Parra FC, Amado RC, Lambertucci JR, Rocha J, et al. (2003). Color and genomic ancestry in Brazilians. Proc. Natl. Acad. Sci. U. S. A. 100: 177-182.

Paskulin DD, Fallavena PRV, Paludo FJO, Borges TJ, et al. (2011). TNF -308G > a promoter polymorphism (rs1800629) and outcome from critical illness. Braz. J. Infect. Dis. 15: 231-238.

Tesauro M, Thompson WC, Rogliani P, Qi L, et al. (2000). Intracellular processing of endothelial nitric oxide synthase isoforms associated with differences in severity of cardiopulmonary diseases: cleavage of proteins with aspartate vs. glutamate at position 298. Proc. Natl. Acad. Sci. U. S. A. 97: 2832-2835.

Umans JG and Levi R (1995). Nitric oxide in the regulation of blood flow and arterial pressure. Annu. Rev. Physiol. 57: 771-790.

Veldman BA, Spiering W, Doevendans PA, Vervoort G, et al. (2002). The Glu298Asp polymorphism of the NOS 3 gene as a determinant of the baseline production of nitric oxide. J. Hypertens. 20: 2023-2027.

Vincent JL, de Mendonça A, Cantraine F, Moreno R, et al. (1998). Use of the SOFA score to assess the incidence of organ dysfunction/failure in intensive care units: results of a multicenter, prospective study. Working group on "sepsisrelated problems" of the European Society of Intensive Care Medicine. Crit. Care Med. 26: 1793-1800. 